FORMATION Formation emploi

Revue française de sciences sociales

128 | octobre-décembre 2014

Pêle-Mêle

\title{
L'injonction au projet chez les jeunes en baccalauréat professionnel : entre soumission et prise en main fragile de son destin
}

The injunction for a project among young people in vocational baccalaureate:

between submission and decision fragile hand of his destiny

Einbindung in ein Berufsprojekt (,injonction au projet") bei Abiturienten des

beruflichen Zweigs : zwischen Fügsamkeit und fragiler Lenkung des eigenen

Schicksals

La conminación al proyecto entre los jóvenes bachilleres técnicos : entre

sumisión y asunción frágil del propio destino

\section{Céline Dagot et Véronique Dassié}

\section{OpenEdition}

Journals

Édition électronique

URL : http://journals.openedition.org/formationemploi/4271

DOI : 10.4000/formationemploi.4271

ISSN : 2107-0946

Éditeur

La Documentation française

Édition imprimée

Date de publication : 31 décembre 2014

Pagination : 7-29

ISSN : 0759-6340

\section{Référence électronique}

Céline Dagot et Véronique Dassié, «L'injonction au projet chez les jeunes en baccalauréat professionnel : entre soumission et prise en main fragile de son destin », Formation emploi [En ligne], 128 | octobre-décembre 2014, mis en ligne le 15 janvier 2015, consulté le 30 octobre 2020. URL : http://journals.openedition.org/formationemploi/4271 ; DOI : https://doi.org/10.4000/ formationemploi.4271 


\title{
L'injonction au projet chez les jeunes en baccalauréat professionnel : entre soumission et prise en main fragile de son destin
}

\begin{abstract}
Céline Dagot
Sociologue, ingénieure d'études au CETU ETICS (Centre d'Etudes Transfert Universitaire Ingénierie, Connaissance Sociale) et chercheure associée au laboratoire Cités, TERritoires, Environnement et Sociétés (CITERES UMR CNRS 7324), université de Tours.
\end{abstract}

VéroniQue DASSIÉ Ethnologue, chargée de recherche à l'Institut d'Ethnologie Méditerranéenne Européenne et Comparative (IDEMEC UMR CNRS 7307), Maison Méditerranéenne des Sciences de I'Homme, université Aix-Marseille.

Résumé

L'injonction au projet chez les jeunes en baccalauréat professionnel : entre soumission et prise en main fragile de son destin

L'injonction au projet invite les jeunes à devenir acteurs de leur avenir. Ils sont contraints à " choisir " une voie. A partir des notions de l'identité repérées par Paul Ricoeur, la " mêmeté " et l'" ipséité » (respectivement présence ou absence de noyau immuable de la personnalité), cet article envisage deux formes de construction narrative et biographique face à l'injonction au projet. Elles vont de pair avec deux formes de réponse à cette injonction qui expliquent le décalage entre les attentes institutionnelles et celles des jeunes en enseignement professionnel.

Mots clés :

Baccalauréat professionnel, projet de formation, orientation scolaire et professionnelle, cheminement scolaire, récit de vie

Abstract

The injunction for a project among young people in vocational baccalaureate: between submission and decision fragile hand of his destiny.

The injunction to have a project invites young people to become involved in their future. They are forced to choose a path. Using the notions of identity defined by Paul Ricoeur, "sameness" and "ipseity" (respectively the presence or absence of immutable core of the personality), this article suggests two forms of narrative construction and biographical faced with the injunction to define a project. Both forms are associated 
with two kinds of answer to this injunction, which explain the gap between institutional expectations and those of young people who receive vocational training.

\section{Keywords:}

Vocational baccalaureate, training project, school guidance \& vocational guidance, school paths, life story

Journal of Economic Literature: I 29

Traduction : Auteures

La notion de projet revêt une dimension importante dans notre société. Elle se propage à tous les pans de vie des individus et à tous les champs, du projet culturel au projet thérapeutique, en passant par le projet de loi, de carrière ou de recherche (Boutinet, 2005). Le champ de l'éducation et de la formation n'échappe pas à cette inflation : le projet d'étude, qu'il soit personnel, de formation, d'orientation, professionnel ou de vie occupe, depuis la fin des années 1980, une place prépondérante dans les réflexions des politiques éducatives et de formation ; ce phénomène s'observe à tous les niveaux de l'enseignement, de la fin du primaire à la formation supérieure.

Aujourd'hui, l'injonction au projet est telle qu'elle y est devenue "une sorte de dogme, avoir un projet serait une condition, sinon nécessaire mais en tout cas décisive pour réussir une démarche de formation-insertion" (Coquelle, 1994).

La notion de projet s'articule ainsi avec les politiques d'emploi et s'inscrit dans le champ plus global des politiques publiques. Le chômage massif des jeunes (Insee, 2013) et le constat récurrent de leurs difficultés d'insertion professionnelle au terme de leur formation ont encouragé diverses réflexions sur la manière de les aider à trouver leur place dans un marché d'emploi de plus en plus sélectif. Pléthore de mesures ont été menées, tant du côté du marché de l'emploi, avec la multiplication des emplois aidés, que du côté de la transformation de l'offre de formation. Sur le plan structurel, elles se sont traduites par l'allongement de la scolarité, la massification de la scolarisation dans le second cycle du secondaire, l'instauration des lycées professionnels (1977), la création du baccalauréat professionnel (1985), l'objectif des 80 \% d'une classe d'âge au niveau du baccalauréat (1989) et la réforme de l'enseignement professionnel en trois ans (2009). Si ces différentes mesures aspirent à l'élévation du niveau de qualification des jeunes, elles tendent aussi à retarder leur arrivée sur le marché du travail.

Dans ce paradigme, l'injonction au projet s'est imposée aux jeunes et est devenue un enjeu essentiel pour les politiques d'éducation et de formation. À l'instar du monde de l'entreprise, le projet a ainsi pénétré ce champ par volonté d'optimiser l'efficacité de l'acte éducatif. 
Apparue officiellement au milieu des années 1970, la notion de projet s'est implantée progressivement dans le cadre scolaire, sous l'impulsion des Projets d'Action Culturelle, Technique et Educative (PACTE, 1979), puis des Projets d'Action Educative (PAE, 1981). En institutionnalisant le projet, la loi d'orientation sur l'éducation de 1989 a révisé le sens de l'acte éducatif en proposant de faire de l'élève l'acteur de sa propre orientation. Les jeunes ne doivent plus subir leur formation mais en être les acteurs, afin d'optimiser leurs chances de réussite, charge à l'institution scolaire de les mobiliser et les motiver. Pour tenir la clé de son insertion future, il incombe alors à chacun d'envisager un projet avant de s'orienter dans une voie scolaire (Prémartin, Legrès, 1988).

Toutefois, de telles préconisations se heurtent à une réalité sociale complexe. L'observation des processus d'orientation révèle en effet d'importantes inégalités et disparités selon l'origine sociale des jeunes. Leur réussite scolaire est fortement corrélée avec leur milieu social.

En fin de $3^{\text {ème }}$, lors du premier palier d'orientation, les élèves et leur famille sont confrontés à un système très hiérarchisé, positionnant au premier rang la voie de l'enseignement général et technologique, au détriment de l'enseignement professionnel (Arrigui, Gasquet, 2010). Ce système classe une grande fraction des jeunes et poursuit sa sélection à l'intérieur de chacune de ses filières, par des spécialités allant des plus prestigieuses aux moins valorisables et n'offrant pas les mêmes perspectives professionnelles.

Dès lors, si le nombre de bacheliers a fortement augmenté, la diversité des baccalauréats n'œuvre pas au profit des mêmes chances de réussite sociale et professionnelle (Prost, 1986 ; Merle, 2002). Au contraire, elle révèle de fortes disparités selon l'origine sociale (Duru-Bellat, Kieffer, 2008 ; Brinbaum, Guégnard, 2012). Les inégalités sociales migrent selon le type de diplôme préparé. Elles s'instituent tant au niveau des performances scolaires, en lien avec le milieu d'origine, qu'au niveau des différences $\mathrm{du}$ choix des filières, en lien avec les aspirations et les autocensures individuelles et familiales (Landrier, Nakhili, 2010).

Parallèlement, des décisions d'orientation sont prises par les acteurs institutionnels (Boudesseul, 2010). Ils valident ou invalident les choix des jeunes et de leur famille selon les performances scolaires, le niveau économique et culturel (Duru-Bellat, Mingat, 1993), mais aussi selon la gestion des flux d'élèves.

Dans ce contexte, l'injonction au projet est une aporie : alors même que l'orientation est " traditionnellement située au confluent des logiques individuelles et des régulations institutionnelles» (Borras, Romani, 2010 ; Berthet et al., 2008), bon nombre d'auteurs révèlent que les politiques d'orientation dans les académies sont très largement régulées par l'offre de formation. Des générations entières sont triées pour alimenter les structures pédagogiques existantes (Van Zanten, 2001 ; Arrigui et al., 2010). Dans cette perspective de gestion des flux d'élèves se joue une distribution des places qui «confere 
aux procédures d'orientation une dimension fortement prescriptive, en particulier pour les jeunes les plus en difficulté" (Berthet et al., 2010).

La hiérarchisation des filières et l'affectation des places conduisent trop souvent encore les acteurs de l'orientation à diriger les élèves les plus en difficulté vers les filières professionnelles, sans prendre en considération leurs goûts et leurs intérêts (Biémar et al., 2003 ; Duru-Bellat, 2002 ). Plus largement relégués dans la voie professionnelle que les jeunes des classes moyennes ou supérieures, ils sont aussi plus souvent soumis à la sélection drastique de cette voie d'enseignement, en raison des places limitées qu'offrent ses spécialités (Grelet, 2006). Ils sont alors contraints à s'orienter de manière aléatoire dans des spécialités en fonction des places disponibles.

Pour toutes ces raisons, bon nombre de lycéens se retrouvent dans la voie professionnelle et sont sommés de conduire un projet dans une spécialité non choisie.

Ce processus d'orientation induit sensiblement une situation paradoxale. Alors que la possibilité de construire un projet en lien avec ses aspirations personnelles est considérée comme la clef de voûte d'une insertion professionnelle réussie, les possibilités d'orientation dans une filière choisie s'avèrent d'autant plus réduites que les jeunes sont issus de milieux sociaux défavorisés. Soumis à l'injonction de faire de leur baccalauréat un maillon de leur " projet professionnel ", gage de réussite et d'insertion sociale, ils ont finalement peu de latitude pour choisir la filière professionnelle dans laquelle ils auraient intérêts et goûts à être formés.

Cet article se focalise donc sur les jeunes issus de milieux modestes, invités dès le collège à construire un projet qui les rendra acteurs de leur devenir, qui se retrouvent malgré eux orientés en baccalauréat professionnel dans une filière non choisie. Contraints à envisager un projet professionnel à court terme, comment répondent-ils à ces injonctions contradictoires? Que l'injonction au projet soit liée au processus d'influence, aux impératifs de gestion des flux d'élèves, ou orientée selon une logique d'adéquation formation-emploi ou d'employabilité, comment la notion de projet est-elle vécue de l'intérieur par ces jeunes? Comment envisagent-ils leur avenir et quels projets font-ils ? Comment la notion de projet contribue-t-elle à leur construction identitaire?

De nombreux travaux traitent des projets de formation scolaire des jeunes et mettent en avant l'enjeu de l'affectation et du diplôme préparé au regard des potentialités d'insertion professionnelle (Boudesseul, Grelet, 2010 ; Arrigui, Sulzer, 2012) ; cependant, les réflexions sur la manière dont ils envisagent la notion de projet dans le cadre d'une orientation "frustrée ", ou "contrariée " ${ }^{(B r i n b a u m ~ e t ~ a l, . ~ 2012) ~ s o n t ~ p l u s ~ r a r e s . ~ L a ~}$

1. Ces auteures s'intéressent rétrospectivement aux parcours de formation et d'insertion des jeunes issus de l'immigration au regard de l'orientation. Elles constatent que l'orientation contrariée, c'est-à-dire l'orientation subie dans des filières peu valorisées et valorisantes, diminue l'obtention d'un diplôme et peut en grande partie expliquer les échecs dans les filières professionnelles, impactant de surcroît l'insertion professionnelle. L'idée est donc d'utiliser cette notion d'orientation contrariée, non pas rétrospectivement, une fois les jeunes 
rencontre avec les lycéens inscrits en baccalauréat professionnel et l'écoute du récit de leurs histoires nous ont permis d'observer des rapports biographiques différenciés qui ont éclairé l'arbitraire des injonctions au récit de soi. Reconstituer leur parcours, fouiller dans leur passé, scolaire et familial, les raisons de leur présence dans un lycée professionnel révélait toute la difficulté, pour ces jeunes, à reconstituer le fil cohérent d'une histoire où s'articulent le passé, le présent et le futur.

Leurs manières de se raconter faisaient ainsi écho aux perspectives de Paul Ricœur à propos d'une identité fondée sur une dialectique de l'idem et de l'ipse. Les différents récits de soi renvoyaient alternativement à la mise en œuvre d'une identité au sens d'idem "dont la permanence dans le temps constitue le degré le plus élevé " et au sens d'ipse, qui "n'implique aucune assertion concernant un prétendu noyau non changeant de la personnalité " (Ricœur, 1990, pp. 12-13). Dans certains cas prédominait ainsi l'idée de fondements d'une permanence de l'être, la "mêmeté ", où les expériences vécues sont suspendues au temps présent, posture qui, par nature, n'est pas sujette au changement mais s'inscrit dans une suite logique de prédispositions fondamentales. Dans d'autres cas prédominait l'idée d'une confrontation de l'être, de l' "ipséité ", où les événements biographiques mis en avant suggèrent la possibilité d'un être changeant, malléable. Les expériences vécues s'articulent, s'unifient et permettent une prise sur sa situation présente et à venir. Cette unification serait la seule garante d'une autonomie.

Or, la première forme, celle d'une identité "du même ", au sens ricoeurien, rend finalement l'idée de projet quelque peu incongrue, compte tenu d'une mise en cohérence identitaire qui, comme nous le verrons, ne passe guère par une linéarisation du discours.

Cet article propose une approche biographique, centrée sur la perception et le discours des jeunes quant à leur parcours scolaire en lien avec leurs aspirations. Il s'agit, par ce procédé, de démêler les nœuds du passé et du futur tels qu'ils s'incarnent dans le vécu des jeunes. Les travaux menés par le philosophe sur la notion d'identité narrative apparaissent particulièrement féconds de ce point de vue. Ils permettent en effet d'envisager la construction discursive des jeunes comme une tentative de réponse formelle à la mise en cohérence d'une identité personnelle et de la confronter à la notion de projet.

Défini comme une "anticipation opératoire " (Boutinet, 2005) d'un futur désiré, le projet est, par principe, une construction dynamique, temporalisée et linéaire, une action en vue d'un changement qui implique une représentation positive de l'avenir. Avoir un projet implique vouloir prendre son destin en main, accéder à l'autonomie et prouver sa capacité à évoluer dans un environnement incertain. Le projet suppose une aptitude à la projection $^{2}$ et à la mobilisation de ressources et de compétences indispensables à sa réalisa-

sortis de formation, à l'entrée de la vie active, mais de manière introspective au moment où les jeunes sont encore en formation dans l'enseignement secondaire.

2. Etymologiquement, le mot projet est un héritage du latin projicere, qui signifie jeter en avant. Comme le 
tion. Son caractère libre, ouvert et non contraint nécessite également que les individus disposent de connaissances suffisantes de leur environnement en lien avec leurs aspirations. En aidant les individus à donner une orientation pourvue de sens, appropriée à leurs aspirations, le projet contribue ainsi au sentiment d'identité. Il amène le sujet à s'assurer de sa continuité, c'est-à-dire à se réaliser comme ayant un passé, un présent et un avenir. Elaboré dans le présent en lien avec les événements passés et futurs, il fait de la sorte appel aux ressorts de l'identité narrative analysée par Paul Ricœur.

À partir d'une analyse qui ne porte pas sur le dispositif d'accompagnement des jeunes mais qui adopte un regard intérieur, celui des jeunes eux-mêmes sur leur formation et leur avenir, notre article se propose d'ouvrir de nouvelles pistes de réflexion.

Il s'agira de saisir la complexité d'un système dans lequel le projet perd finalement une partie de son efficacité dans la corrélation formation-emploi, en raison d'une modalité de construction narrative implicite au projet qui ne va pas de soi chez les jeunes ( $c f$. encadré 1). Nous rendrons ainsi compte de deux polarités de construction narrative qui encadrent la possibilité de faire des projets en relation avec des manières de se raconter et de concevoir sa propre identité. La première forme, exposée en première partie, concerne des jeunes "sans projet " qui se pensent au présent, ce qui les empêche d'établir des relations de causalité, par conséquent d'agir au nom d'un projet. La seconde forme, abordée en seconde partie, rend compte de jeunes qui élaborent des " contre-projets ", révélant alors la propension à objectiver des contraintes et à «tenir " son propre destin en main, situation propice à l'élaboration d'un projet d'avenir.

Au fil de ces deux parties, révélant finalement que les jeunes ne se construisent pas véritablement avec l'institution mais plus à côté ou contre elle, l'idée est de scruter de plus près les enjeux d'une telle construction identitaire. Il s'agit de saisir de quelle manière ils se projettent sur le plan scolaire et professionnel, et plus largement dans leur vie quotidienne.

C'est donc toute la question de la fabrique d'un " je suis " face à l'impératif d' "être soi» (Ehrenberg, 1995) mais aussi l'auto-construction narrative du biographique face à l'injonction au projet qui seront ici questionnées.

rappelle Alain Rey, l'élargissement sémantique du terme remonte à la Renaissance et résulte de son emprunt dans le vocabulaire de l'architecture : pour réduire la part d'improvisation de leurs réalisations techniques, les architectes réalisent des maquettes permettant de concrétiser et de matérialiser une intuition, avant de la réaliser en grandeur nature. Dès lors, la notion de projet implique l'idée d'une réalisation à la fois spatiale et temporelle qui sépare la conception, élaborée en amont, de sa réalisation (Rey, 1995). 


\section{Encadré 1 : Méthodologie}

Les matériaux mobilisés ici sont tirés d'une enquête collective réalisée dans le cadre d'un Appel à Projet Recherche de la région Centre, intitulée DevenirActiflci, portée par le laboratoire CITERES (Cités, TERritoires, Environnement et Sociétés). L'objectif de cette recherche visait à saisir le sens et la perception qu'ont les jeunes de leur avenir, notamment en termes de perspective professionnelle et plus largement en termes de projet de vie. Il s'agissait de reconstituer leur parcours scolaire en l'articulant avec les dimensions familiales, sociales, institutionnelles et territoriales. 60 entretiens semi-directifs ont été réalisés entre septembre 2012 et janvier 2013, auprès de jeunes inscrits en baccalauréat professionnel ; 30 étaient en formation au moment de l'enquête et 30 autres avaient démissionné en cours d'année depuis moins d'un an.

Cinq lycées publics d'enseignement secondaire, situés dans trois départements (Indre, Indre et Loire et Loiret) de la Région Centre ont fait l'objet de nos investigations.

Les baccalauréats professionnels suivis par les jeunes sont diversifiés : Accueil ; Commerce ; Métiers de la mode ; Gestion administration ; Secrétariat ; Comptabilité ; Vente ; Maintenance des équipements industriels; Electrotechnique ; Accompagnement Soins et Services à la Personne ; Médiation/Service de proximité et vie locale.

En concertation avec les proviseurs, les CPE (conseillers principaux d'éducation) ou les enseignants étaient chargés, à partir d'un courrier que nous avions rédigé, d'expliquer notre enquête aux élèves de terminale et de recenser les volontaires. Le panel des lycéens en formation s'est donc constitué sur la base du volontariat et les entretiens ont été réalisés dans l'enceinte des établissements (mise à disposition de bureaux). S'agissant des lycéens ayant démissionné de l'établissement durant l'année scolaire, leurs noms et coordonnées nous ont été communiqués par les chefs d'établissement concernés. Nous avons ciblé, dans un premier temps, les élèves de terminale, puis, compte tenu des difficultés rencontrées (numéros de téléphone plus attribués, déménagement, refus, rendez-vous non honorés) pour atteindre l'effectif visé, nous avons élargi notre population aux élèves de première, puis de seconde. Les entretiens se sont déroulés au domicile des jeunes ou à l'extérieur (café, gare, etc.).

Il importe de préciser que la démission d'un lycéen ne signifie pas mécaniquement la fin des études. Certains ont changé de lycée pour des questions de proximité géographique et continuent dans la même spécialité ; d'autres ont transité vers l'apprentissage, d'autres réalisent une formation dans le cadre de la Mission Locale, etc. Ainsi, les chemins pris par les jeunes à la suite de leur démission sont multiples et variés. La distinction entre les deux populations semblait pertinente pour appréhender la question de l'avenir en termes de perspective professionnelle ; toutefois, elle s'est avérée finalement relative en raison des différents chemins empruntés par les jeunes.

Pour toutes ces raisons, l'examen de la perception d'un avenir " positif » en termes de perspective professionnelle semble important, non pas tant du côté des parcours scolaires linéaires, mais bien plus du côté de l'orientation et de la capacité des jeunes à se l'approprier par le biais d'un projet, processus par lequel se construit une certaine identité narrative.

Pour cet article, les données qualitatives portent exclusivement sur les entretiens réalisés par les auteurs (32) et parmi ce panel, ne sont concernés que les jeunes qui relatent une orientation subie (22) en baccalauréat professionnel. II peut donc s'agir de jeunes toujours en formation en Terminale comme de jeunes ayant démissionné de l'un des cinq lycées enquêtés et qui se retrouvent après coup dans un autre lycée, en formation, en apprentissage, ou sans activité, etc. Tous les jeunes interrogés ne sont pas cités dans cet article ( $c f$. tableau 1). 
Tableau 1 : Caractéristiques sociales des jeunes cités dans cet article

\begin{tabular}{|c|c|c|c|c|c|c|c|c|}
\hline Prénom & Age & Sexe & $\begin{array}{l}\text { Orientation } \\
\text { contrainte }\end{array}$ & $\begin{array}{c}\text { Orientation } \\
\text { souhaitée }\end{array}$ & $\begin{array}{c}\text { Classe(s) } \\
\text { doublée(s) }\end{array}$ & $\begin{array}{l}\text { Situation } \\
\text { parentale }\end{array}$ & $\begin{array}{l}\text { PCS Père- } \\
\text { Mère }\end{array}$ & $\begin{array}{l}\text { Situation au } \\
\text { moment de } \\
\text { l'entretien }\end{array}$ \\
\hline Amélie & 17 & $\mathrm{~F}$ & Bac Pro Mode & $\begin{array}{c}\text { Bac pro } \\
\text { Photographie }\end{array}$ & Aucune & Divorcé & $\begin{array}{l}\text { Ouvrier/ } \\
\text { Employée } \\
\text { (boucherie) }\end{array}$ & Terminale Mode \\
\hline William & 18 & H & $\begin{array}{l}\text { Bac Pro } \\
\text { Electrotechnique }\end{array}$ & Bac général & $4^{\text {ème }}$ & Divorcé & $\begin{array}{l}\text { Ouvrier/Agente } \\
\text { Territoriale } \\
\text { Spécialisée Ecole } \\
\text { Maternelle }\end{array}$ & $\begin{array}{c}\text { Terminale } \\
\text { Electrotechnique }\end{array}$ \\
\hline Jérémy & 18 & $H$ & $\begin{array}{l}\text { Bac Pro Maintenance } \\
\text { Equipements } \\
\text { Industriels (MEI) }\end{array}$ & Bac général & $6^{\text {ème }}$ & Union & $\begin{array}{l}\text { Ouvrier/ } \\
\text { Employée } \\
\text { (Mairie) }\end{array}$ & Terminale MEI \\
\hline Laurine & 15 & $\mathrm{~F}$ & Bac Pro Accueil & Bac général & Aucune & Union & $\begin{array}{l}\text { Ouvrier retraité/ } \\
\text { Employée } \\
\text { (ménage) }\end{array}$ & $\begin{array}{l}\text { Démission en } \\
\text { seconde. Pas de } \\
\text { prise en charge. }\end{array}$ \\
\hline Marine & 16 & $\mathrm{~F}$ & Bac Pro Médiation & $\begin{array}{l}\text { Apprentissage } \\
\text { Petite enfance }\end{array}$ & Aucune & Divorcé & $\begin{array}{c}\text { Employé/ } \\
\text { Employée } \\
\text { (boulangerie) }\end{array}$ & $\begin{array}{c}\text { Démission en } 1^{\text {ère }} \text {. } \\
\text { Apprentissage } \\
\text { vente }\end{array}$ \\
\hline Mia & 19 & $\mathrm{~F}$ & Bac Pro Mode & Bac général & CE2, 6 ème & Divorcé & $\begin{array}{c}\text { Ouvrier/Mère au } \\
\text { foyer }\end{array}$ & Terminale Mode \\
\hline Youssa & 18 & $\mathrm{~F}$ & Bac Pro Commerce & $\begin{array}{c}\text { Bac pro } \\
\text { Comptabilité }\end{array}$ & $4^{\text {ème }}$ & Union & $\begin{array}{c}\text { Ouvrier/Mère au } \\
\text { foyer }\end{array}$ & $\begin{array}{c}\text { Démission 1ère } \\
\text { Comptabilité } \\
\text { (déménagement). } \\
\text { Réorientation en } \\
1^{\text {ère }} \text { Commerce } \\
\end{array}$ \\
\hline Joanna & 17 & $\mathrm{~F}$ & Bac Pro Accueil & Bac général & Aucune & Divorcé & $\begin{array}{l}\text { Agriculteur/ } \\
\text { Ouvrière }\end{array}$ & Terminale Accueil \\
\hline Tarik & 18 & $H$ & Bac Pro Comptabilité & $\begin{array}{l}\text { Bac général puis } \\
\text { BTS Commerce }\end{array}$ & CE2 & Séparé & $\begin{array}{l}\text { Commerçant (au } \\
\text { Maroc)/Mère au } \\
\text { foyer (maladie) }\end{array}$ & $\begin{array}{c}\text { Terminale } \\
\text { Comptabilité }\end{array}$ \\
\hline Gloria & 18 & $\mathrm{~F}$ & Bac Pro Secrétariat & Danseuse & Aucune & Divorcé & $\begin{array}{l}\text { Employé/Sans } \\
\text { profession }\end{array}$ & $\begin{array}{l}\text { Terminale } \\
\text { Secrétariat }\end{array}$ \\
\hline
\end{tabular}

\section{La fabrique des sans-projets : des parcours sans linéarité}

Relier un projet à un cursus de formation amène à envisager les contraintes qui pèsent sur son élaboration. Il s'agit en effet pour les jeunes de mettre en œuvre des ressources qui leur permettront d'accéder à une vie professionnelle en lien avec leurs centres d'intérêt. Or ces jeunes revendiquent rarement le désir d'exercer le métier auquel leur formation actuelle les prépare.

Considérant d'abord l'impasse dans laquelle beaucoup se trouvent suite à une orientation subie, nous verrons comment ces jeunes s'en accommodent en faisant du baccalauréat une 
ligne de mire, sans pour autant en faire l'enjeu d'un projet professionnel, ni même plus largement identitaire. Installés dans le présent, la perception de leur parcours et de leur avenir dévoile aussi une absence de linéarité, peu propice à l'élaboration de projets.

\subsection{L'intériorisation d'une orientation par défaut}

Dans la mise en récit, un des points sensibles concerne les conditions d'accès à la formation. Ces jeunes, issus de familles modestes, justifient leur inscription en baccalauréat professionnel par une orientation par défaut. Ils n'ont en effet pas choisi la formation dans laquelle ils sont inscrits et envisagent leur présence dans l'établissement comme une contrainte acceptée.

Ce constat n'est pas nouveau (Daverne, 2012), mais de lui découle un rapport à l'identité narrative qu'il importe de préciser. Selon Ricœur, la conscience d'être soi passe par la possibilité de faire le récit de sa propre histoire en articulant entre eux des événements dissociés dans le temps, d'où la notion d'identité narrative : "Le temps devient humain dans la mesure où il est articulé de manière narrative " (Ricœur, 1990, p. 17). Dès lors, dans le récit qu'il produit sur lui-même, le jeune doit donner du sens à son passé pour être en continuité avec son présent.

Or, faute de bonnes performances scolaires, d'un comportement adapté ou d'une idée d'avenir professionnel, ces lycéens sont contraints de se laisser guider par les acteurs scolaires et peinent à donner du sens à leur situation actuelle :

"C'est ma prof. principale qui m'a dit "de toutes façons, je ne te vois pas aller en générale et tout ça! Il va falloir que tu songes à un parcours professionnel.” (...) Moi j’ai dit oui, je ne savais même pas de quoi on me parlait! (...) C'est eux qui savaient mieux que moi, c'est eux, les professeurs, qui savent ce que je peux faire! " (Mia);

"On m'a refusé l'entrée en enseignement général parce qu'on m’a dit que je n'avais pas les notes pour! (...) Il y a une feuille où il faut formuler des voux pour l'orientation et j'avais marqué enseignement général et ma prof principale m'a dit: "Il faut que tu refasses tes voux parce que tu ne seras jamais acceptée!", donc voilà. Après je suis retournée la voir dans son bureau et elle m'a dit "non, le seul truc que tu peux faire, c'est soit un CAP, soit un Bac Pro". Il fallait reformuler d'autres voux (...) Je me suis dit, si je dois faire un bac pro, je ferai un bac pro vente, sauf qu'au final il n'y avait plus de place en vente, donc ils m'ont mis en accueil " (Laurine).

Mia, Laurine et bien d'autres se trouvent confrontés, au moment de leur $3^{\text {ème }}$, à la hiérarchisation des voies d'enseignement. Alors qu'elles aspiraient à entrer dans l'enseignement général, leur professeur principal a tranché et acté leur incapacité à entrer dans cette voie. Parallèlement, Amélie, qui envisage l'enseignement professionnel en ayant une idée précise du métier qu'elle souhaiterait exercer, voit tout autant ses aspirations remises en cause :

"En fin de 3"ime, je voulais faire une formation de photographe. Sauf qu'à cause de mes notes, j’ai été refusée. Je voulais redoubler pour partir sur de bonnes bases, enfin pour intégrer mon école, 
mais ils m’ont dit que ça ne servait à rien. Donc, du coup, ils mont dit [la proviseur adjointe] "Il y a une formation accueil aux Charmilles."; Donc, du coup, je me suis retrouvée-là. Mais il n’y avait plus de place en accueil, donc je me suis retrouvée en Mode " (Amélie).

Dans cette affectation des places, ces jeunes subissent plus une formation qu'ils ne la choisissent. Mais en plus, les connaissances et les possibilités qu'offrent les spécialités dans lesquelles on les "expédie " leur font également défaut. L'absence de prise sur ce qu'il leur arrive instaure d'emblée un fossé entre leurs aspirations et les cursus proposés, d'autant que ces élèves cumulent des résultats scolaires jugés insuffisants et de faibles ressources familiales et économiques.

Toutefois, si ces jeunes expriment facilement le peu de marges de manœuvre dont ils disposent, ils n'évoquent pas la responsabilité de circonstances extérieures, ni même les conséquences futures de telles décisions. Ils ont pris acte d'un impossible qui justifie une orientation dont ils ne remettent pas en cause la pertinence, puisqu'elle est portée par des acteurs à l'évidence mieux placés qu'eux pour cerner leurs potentialités au regard d'une offre de scolarité.

Cette impossibilité repose ainsi sur une incapacité intrinsèque mise en évidence à travers des notes et des appréciations des enseignants : "désinvolture ", "aucun travail ", "passivité ", peut-on lire, tel un leitmotiv, dans leurs dossiers scolaires. Ces jugements tendent à révéler qu'ils n'ont pas les dispositions requises pour poursuivre des études. Or, penser leur avenir à travers l'idée de "dispositions ", pour ne pas dire de " pré " dispositions (Lahire 2005), revient à établir des propensions à l'agir "permanentes, généralisables et transposables quelle que soit la situation [et à éliminer] le rôle du contexte " (Lahire, 2001, p. 67).

Leur marge de manœuvre apparaît d'emblée réduite, non parce que des circonstances extérieures ne leur ont pas permis de s'engager vers d'autres voies mais parce que leurs aptitudes propres ne le leur permettent pas. Ils ont pris acte d'une identité d'élève qui s'est révélée au collège. C'est donc à travers une expérience de leur "mêmeté" (Ricœur, 1990), c'est-à-dire d'un noyau stable de leur être, telle qu'elle leur est renvoyée par leurs enseignants, qu'ils prennent le chemin que l'institution scolaire trace pour eux.

\subsection{Avoir le baccalauréat : un sésame dans un marché de dupes ?}

L'orientation contrainte n'est toutefois pas forcément synonyme d'échec chez ces jeunes. Face aux injonctions scolaires, beaucoup souhaitent obtenir leur baccalauréat, voire même poursuivre leurs études.

La difficulté majeure, somme toute de taille dans une logique de formation/insertion, c'est que rares sont ces jeunes qui envisagent d'exercer un métier en lien avec leur formation professionnelle. L'attrait du métier est laissé de côté au profit du baccalau- 
réat qui en devient l'horizon ultime (Daverne, 2012). William marque ainsi son peu d'intérêt pour le métier auquel il est formé :

W. : « Le bac, c'est tout ce qui m’intéresse.

$Q:$ :Ta priorité, c'est le bac?"

$W:$ : Oui, pour travailler. »

$Q$ : "Et tu envisages de travailler dans quel secteur?"

W. : "Ben, là où je trouverai, le premier emploi, je prends. Electrotechnique ou pas, ça m'est égal, je prendrai ce qu'il y aura! Si je peux faire un autre métier, même si ce n'est pas électrotechnique, ça passera avec ce Bbc, car ça ouvre pas mal de choses!".

L'objectif se concentre donc sur l'obtention d'un baccalauréat, peu importe lequel, ce diplôme étant considéré comme une sorte de sésame à l'entrée dans le monde du travail. Jérémy explique ainsi avoir pioché dans la liste des baccalauréats disponibles, "pour avoir un bac", parce qu'il faut bien en avoir un : "On n'a pas le choix aujourd'bui !". Ces lycéens ont donc fortement intériorisé le caractère obligatoire de la formation, et plus encore du baccalauréat. Leur scolarité reste valorisable car elle est gratifiée par un diplôme plébiscité.

Or diverses recherches (Beaud, 2002 ; Arrigui, Gasquet, 2010 ; Arrigui, Sulzer, 2012) montrent que le type de baccalauréat conditionne fortement l'insertion professionnelle future et peut s'avérer peu rentable s'il est dissocié de toute finalité professionnelle concrète. La multiplication des baccalauréats dissimule, à bien des égards, des inégalités dont les jeunes n'ont pas vraiment conscience ; dans ces conditions, le diplôme n'est qu'un leurre, un simulacre.

Labsence de lien entre leur formation et leur future activité professionnelle ne tient pas seulement à une erreur d'aiguillage. Faute de pouvoir accueillir tous les élèves dans les formations prisées, l'institution scolaire tend elle-même à minimiser le décalage entre un débouché et l'inscription dans une filière donnée.

Amélie, inscrite en terminale « Métiers de la mode » évoque ainsi son « bac couture ». Elle explique cette redéfinition :

"J'appelle ça couture, mais c'est mode, oui, ce n'est pas particulièrement de la mode, pas du tout même (...) On ne dessine pas des vêtements, on fait un truc imposé, en fait, c'est industriel».

Peu informée en amont du contenu véritable de la formation, elle dit être arrivée « comme ça, parce que ça ne m’intéressait pas déjà!". Elle précise qu'elle n’envisage pas de travailler "dans une entreprise à faire de la couture! " mais ne remet pas pour autant en cause l'inadéquation de l'intitulé du diplôme, car seul le passeport du bac lui importe pour ses projets :

"Après, je voudrais entrer dans une fac des beaux-arts pour me perfectionner au niveau dessin car plus tard j'aimerais être tatoueuse! J'aimerais bien faire ça!" 
Q. : "D'accord, ça n'a rien à voir avec ta formation actuelle?"

A. : "Non, non, rien à voir. Il n'y a pas du tout de lien et ça ne mintéresse pas!"

À ces logiques contradictoires, les jeunes répondent par un bricolage, au sens où l'a envisagé C. Lévi-Strauss (1962, p. 31). En tant que bricoleur, leur "univers instrumental est clos et la règle du jeu est de toujours sarranger avec les moyens du bord ", ce qui différencie leur mode d'action de celui de l'ingénieur dont "les tâches exécutées sont subordonnées à l'obtention de matières premières et d'outils conçus et procurés à la mesure de son projet " (Ibid.).

L'orientation contrainte amène donc ces jeunes à se raccrocher à l'enjeu du diplôme et à dissocier leur formation du métier préparé. Ils apprennent des savoir-faire qu'ils n’envisagent pas d'utiliser plus tard, ce qui complexifie la possibilité d'établir un lien entre leur présent et leur avenir. Il leur est également difficile d'articuler les éléments du passé avec le futur, ce qui renforce le vécu d'une identité essentialiste et pérenne d'un " même » incorporé et, par contrecoup, "occulte son ipséité " (Ricœur, 1990, p. 46). Faute de pouvoir se vivre à travers une forme d'ipséité, ces jeunes n'envisagent pas de changer l'état permanent d'une identité immuable pour accéder à une nouvelle identité. Le métier qu'ils préparent ne coïncide pas avec l'idée qu'ils ont d'eux-mêmes, d'une identité qui leur est propre et fondée sur le principe d'un noyau inaltérable et immuable. Dans ces conditions, la notion de projet est réduite à l'obtention d'un diplôme qui ne s'articule pas avec un après.

\section{3. Un présent sans passé ni avenir}

Détachée de leur propre existence en termes d'actions, de motivations et de projections, la scolarité de ces élèves est donc vécue comme un présent contraint auquel il serait vain de vouloir échapper. Ils disent être là parce qu'il leur faut être là, parce qu'“ $O n$ ", disent-ils, sous-entendu un ou des adultes, leur a dit d'être là.

Ils vivent leur scolarité au jour le jour, sans véritablement formuler de projection dans l'avenir, ni même repérer de traces la reliant au passé. Diffus, éclaté, désarticulé dans le temps, leur cursus se trouve détaché de leurs aspirations professionnelles. Les jeunes éprouvent par conséquent des difficultés à envisager leur scolarité comme un cheminement continu, fut-il fait de bifurcations, de discontinuités et de ruptures (Bidart, 2006 ; Coineau, Vivent, 2010). Ils ne conçoivent pas de liens entre ce qui leur arrive en termes de scolarité et les événements extérieurs, passés ou à venir, qu'ils soient familiaux, institutionnels, économiques ou territoriaux.

L'absence d'articulation entre les expériences n'a pas pour corollaire une absence d'événements vécus. Beaucoup de ces jeunes ont été confrontés à des problèmes de santé, des séparations familiales, des mobilités géographiques ou encore des situations nécessitant l'intervention de travailleurs sociaux, voire de la justice.

De tels bouleversements sont décrits par la psychosociologie comme des freins à la mise en œuvre d'un projet, "l'idée même de construire un projet professionnel [a] peu de signification 
en soi tellement ils se trouvent débordés par des problèmes de survie au quotidien " (Fournier, 2002, p. 380). Pourtant, le tragique qui affleure dans certaines histoires se raconte sans en avoir l'air, comme la déclinaison particulière de n'importe quelle histoire familiale, rendant impensable l'expression d'une inégalité sociale qui le détermine par ailleurs.

L'histoire d'Amélie illustre la manière dont certains jeunes envisagent leur propre histoire. Plutôt bonne élève en primaire : "jétais dans les cinq meilleurs élèves de ma classe en primaire ", Amélie voit subitement ses résultats scolaires chuter lors de son entrée au collège. Elle ne fournit pas d'explication à cette baisse, si ce n'est qu'elle était « un peu perturbatrice " et " bavarde" :

Q. : "Et comment tu expliques la baisse de tes résultats par rapport à ta primaire?"

A. : "Ben je ne faisais rien, juste le minimum, jeetais un peu enquiquineuse et puis bavarde aussi!" (Plus loin dans l'entretien) : "Mon père a perdu son emploi et il s'est mis à boire, et c'est de là que des comédies pas possibles entre mes parents ont commencé jusqu'à ce que ma mère divorce l'année dernière (...)"

Q. : "Et quand ton père s'est mis à boire, tu avais quel âge?

A. : "Oh, jétais petite, ceetait à la fin de mon CM2, je devais avoir 11 ans!".

Amélie évoque cette histoire familiale sans établir de lien avec sa scolarité, comme si l'une et l'autre se déroulaient en parallèle sans jamais se croiser. Pourtant, la chute de ses résultats coïncide avec le moment où son père "s'est mis à boire ", suite à la perte de son emploi, et que des scènes de ménage ont commencé à surgir dans le foyer.

Joanna, inscrite en terminale bac pro Accueil, est dans la même posture qu'Amélie. Après une année de maternelle à Eguzon, dont elle ne garde aucun souvenir, elle change d'école. Elle déménagera à nouveau au moment du collège. À l'issue du collège, elle dit vouloir continuer ses études pour obtenir un bac général mais ses résultats sont insuffisants. Elle envisage donc de s'inscrire en baccalauréat professionnel à Bourges, "parce que c'est plus loin ", précise-t-elle, comme si seule la motivation à s'extraire d'un contexte local importait à ses yeux, sans aller plus loin pour expliquer ce désir d'éloignement. Mais son dossier n'est pas retenu. Elle reste donc à Châteauroux et entame une seconde Accueil au lycée des Charmilles, "le seul établissement qui l'accepte!". Interrogée à propos de ses projets, elle pense obtenir son bac mais ne sait pas ce qu'elle fera l'an prochain. Elle rêve de vivre à New York mais n'anticipe aucun dispositif concret pour réaliser ce rêve. Seul l'espoir d'être embauchée pendant les vacances comme caissière lui permet d'espérer passer des vacances d'été avec des amis à Ibiza. En parallèle de sa scolarité, des événements familiaux, divorce, recomposition familiale, jalonnent son histoire, sans qu'elle les relie davantage les uns aux autres. Elle ne les aborde d'ailleurs jamais spontanément. De manière générale, elle n'évoque sa famille qu'avec réticence et relate peu de souvenirs familiaux. Ses proches ne sont pourtant pas absents de sa scolarité. Joanna continue en effet ses études parce que son père lui a dit : "Il faut que tu continues!". 
Si les lacunes et les résistances mémorielles compliquent la mise en récit, elles rendent également difficile toute articulation entre les expériences vécues. Or, c'est bien par le biais du souvenir que l'individu nourrit une identité narrative qui se révèle dans la "dialectique de l'ipséité et de la mêmeté " (Ricœur, 1990, p. 167). Les événements s'enchaînent dans l'histoire des jeunes sans qu'ils aient réellement de prise sur eux. Régulièrement invités à se projeter et à mettre en œuvre des ressources qui leur permettront de s'insérer dans le marché de l'emploi, ils se trouvent dans des situations où les expériences vécues apparaissent totalement dissociées les unes des autres. L'absence de liants, d'enchevêtrements, ne favorise pas la mise en forme d'un discours rétrospectif et/ou prospectif, mais alimente au contraire un discours de la présence au temps présent.

Youssa, inscrite dans un premier temps en bac pro Comptabilité, projet très largement mené dans le cadre de sa $3^{\text {ème }}$ DP6 (option découverte professionnelle, six heures), se voit contrainte, suite au déménagement de ses parents, de se réorienter en première Commerce, la seule filière où il restait de la place. Elle conclut alors à propos de sa scolarité : "J'ai rien à dire, j'en pense rien, j'avance au jour le jour, c'est tout!».

Ces propos posent la question du caractère passif de leur position d'élèves, mineurs, et de l'absence de maîtrise de leur propre avenir. Si ces jeunes n'ont pu décider de leur formation, ils ne se réapproprient pas pour autant leur vécu pour en faire leur histoire, dont ils seraient dignes de témoigner.

Ce non-positionnement dans une temporalité historique révèle la difficulté que ces jeunes éprouvent à donner du sens à un présent face à un avenir qui semble déjà tracé. Ils montrent ainsi leur propension à accepter, à s'effacer, et à évacuer toute idée de déterminisme social mais aussi toute logique d'action contraire. Si le projet peut être assimilé à un effort "qui pousse le Moi en dehors de Soi à partir d'une action réciproque entre le Moi et quelque chose d'extérieur à celui-ci " (Boutinet, 2005, p. 24), il ne peut prendre forme ici faute d'articulation des événements vécus. Cette posture joue en faveur d'une propension à l'identité du "même " (Ricoeur, 1990) sur laquelle l'extériorité n’a finalement que peu de prise. Ces jeunes sont ainsi dans l'incapacité d'élaborer un projet professionnel prescrit par l'extérieur, notamment l'institution scolaire. L'injonction au projet est un non-sens.

\section{La fabrique d'un contre-projet: 2 une mise en intrigue du biographique}

Contraints de subir une formation et soumis au même contexte social que les jeunes précédents, d'autres jeunes évoquent toutefois un avenir professionnel qui se dessine comme un horizon concret en vue duquel ils élaborent une stratégie d'action. Précisons toutefois que ces jeunes expriment des formes de rébellion contre des étiquetages scolaires et agissent paradoxalement au prix d'un pas de côté vis-à-vis des propositions de l'institution scolaire. 
Ces élèves révoltés mettent cependant en œuvre une construction narrative qui se différencie de celle des jeunes évoqués précédemment. S’ils peuvent envisager leur passé et leur avenir, c'est au prix d'une remémoration propice à l'élaboration d'une linéarisation biographique, c'est-à-dire d'une mise en intrigue où " des buts, des causes, des hasards sont rassemblés sous l'unité temporelle d'une action totale et complète " (Ricoeur, 1983, p. 10). Cette construction semble favoriser la possibilité de prendre prise sur un avenir. Il reste à voir comment cette remémoration peut devenir un outil au service d'un projet véritablement personnel, quand le projet élaboré se dessine contre l'institution.

\subsection{De l'injonction au projet personnel à la reproduction sociale}

Déceler l'appartenance à un milieu " défavorisé » comme la source de difficultés scolaires reviendrait à établir un jugement négatif sur sa propre famille. Or, aussi compliqué soitil, le vécu quotidien n'en est pas moins rempli d'affectivité par celui qui le vit. L'histoire familiale et l'histoire scolaire ne sauraient en conséquence être superposables. Établir une relation de cause à effet entre les deux s'avère donc difficile.

Si pour les lycéens évoqués en première partie, la dissociation des sphères privées et scolaires s'accompagne d'une intériorisation, d'une impossibilité d'aller contre la voie qui leur est pour ainsi dire imposée, en les rendant imperméables aux injonctions scolaires, d'autres adoptent au contraire une posture moins passive. Aux côtéx des résignés, il y a également les rebelles, qui n'acceptent pas leur situation et tentent de s'y opposer.

L'histoire de Tarik, inscrit en terminale Comptabilité, est exemplaire de ce point de vue. À l'âge de sept ans, il est envoyé au Maroc chez son père qu'il ne connaît pratiquement pas. Sa mère, malade, ne peut plus s'occuper de lui. De 7 à 13 ans, il alterne les séjours entre la France et le Maroc avant de revenir définitivement chez sa mère. Au Maroc, il découvre un pays étranger et une famille inconnue, dont une petite sœur. Inscrit dans un lycée privé français, il dit "ne pas travailler" et "faire n’importe quoi " et arrête finalement l'école. Pour justifier cet arrêt, il explique simplement l'absence d'envie de travailler, " comme tout enfant", même si cette rupture résulte surtout de frais de scolarité impayés. S’il dit avoir eu "les nerfs", sa colère n'est toutefois pas dirigée contre sa famille, ni contre un contexte social et affectif difficile, "y'a pire ", mais contre l'institution scolaire "la prof qui m'aimait pas» et "l'école » qui l'a mis à la porte.

Les événements familiaux ne sont toutefois pas appréhendés comme étant à l'origine de sa mise au ban par l'école. Tarik met au contraire en avant la normalité de sa situation : comme tout enfant, il préfère s'amuser et aime ses parents. C'est pourquoi le responsable désigné pour justifier des difficultés scolaires s'incarne en la personne d'un représentant de l'institution scolaire. Comme le remarque également P. Ricœur à propos des justifications causales d'une action, les élèves procèdent ainsi sans véritablement établir de lien entre le comment et le pourquoi de leurs actions. Justifié en termes affectifs (quelqu'un ne m'aimait pas), le départ de l'école implique un objet (l'enseignant) comme cause et "une 
certaine passivité sávère corrélative à l'action de faire " (Ricœur, 1990, p. 84). L'événement déclencheur du rejet de l'école implique une relation interindividuelle vécue sur un mode négatif. Ce faisant, l'individu étiqueté déficient sur le plan scolaire produit un discours qui rétablit sa propre normativité face à une expérience de stigmatisation. Inversant les approches déterministes qui pointent l'importance du milieu social dans la production des difficultés scolaires, certaines approches interactionnistes tendent également à imputer à l'individu-enseignant la responsabilité de l'éloignement d'un élève de l'école (EsterleHedibel, 2007 ; Douat, 2011). Un tel transfert de responsabilité occulte néanmoins la mécanique structurelle qui le sous-tend : au-delà de l'événement déclencheur et explicatif du rejet de l'école se joue en effet l'incompatibilité entre l'injonction au projet, portée par l'institution, et les projets nourris par des élèves ou leur famille.

Faute de pouvoir lutter contre son sort, Tarik entre donc en rébellion contre l'institution scolaire. Son comportement lui vaut d'être rejeté de la filière générale à laquelle il aspire pour se retrouver en bac pro Comptabilité, dans une classe de «cas soc » dit-il. Sa demande de bac Pro Commerce est également refusée, alors même que celle d'un de ses camarades aux performances scolaires moindres est acceptée. Tarik pointe ainsi des exemples qui ont valeur d'injustice et qui justifient d'autant plus sa colère à l'égard du système scolaire. Son projet, faire du commerce, est désormais totalement dissocié d'une stratégie scolaire à laquelle il se prête de mauvais gré.

La rébellion peut toutefois revêtir des formes moins conflictuelles vis-à-vis de l'école. Marine, 17 ans, voudrait travailler avec des enfants, mais à l'issue de sa $3^{\text {ème }}$, sa mère, peu informée des codes et des stratégies scolaires, "s'est trompée " en l'inscrivant en médiation au lycée professionnel. Arrivée en classe de $1^{\text {ère }}$, elle décide "sur un coup de tête " d'arrêter sa scolarité :

"Le matin, je miennuyais tellement que l'après-midi, je n'ai pas été en cours, j’ai fait toutes les rues de la ville de Tours et j'ai trouvé un patron... ça faisait quelques mois que jen avais parlé à ma mère, mais elle ne voulait pas, mais après, vu que j’ai trouvé un patron, elle m'a dit oui! Pour elle, un bac pro, c'était mieux, enfin c'est plus intéressant, plus valorisé (...) Mon père, jen ai parlé que quand la patronne m'a vraiment dit oui et il l'a encore moins bien pris que ma mère en fait!"

La référence au " coup de tête " établit une rupture franche entre un avant et un après et enclenche la mise en œuvre de démarches qui marquent son rejet d'une formation envisagée pour elle. Elle décide d'une autre voie que celle initialement prévue et agit pour concrétiser son projet hors de son lycée, malgré les résistances familiales et leurs conséquences ; son père ne lui adressera plus la parole pendant plusieurs jours. Elle trouve ainsi une place d'apprentie en boulangerie et s'inscrit en CAP (certificat d'aptitude professionnelle) dans un CFA (centre de formation des apprentis). La déception de ses parents cède alors la place à la satisfaction : "ils étaient contents parce que je me suis débrouillée toute seule ». L'abandon du diplôme et la déqualification sociale qui pourrait en résulter apparaissent compensés par la preuve de son autonomie. La rupture avec l'école enclenche un regard rétrospectif 
qui autorise le regard prospectif et, ce faisant, intervient dans l'ébauche d'une linéarisation du biographique et de la construction d'un projet d'avenir. Marine n'évoque pas la déqualification liée à un choix, qui la conduit insidieusement davantage dans les pas de sa mère elle-même vendeuse dans une boulangerie- que vers ses rêves de puériculture. L'ipséité est ainsi mise au service d'une mêmeté qui, par ce contre-projet, décline le noyau dur d'une identité vécue autant au niveau de la sphère individuelle que socio-familiale. Alors que P. Geminel (1988) voyait dans le projet un gage d'autonomie et d'investissement professionnel, il s'avère ici d'une portée relativement illusoire. L'interruption de sa formation pour travailler dans une boulangerie montre que sa volonté d'aller vers l'imprévu pour prendre en main son avenir (rapport à l'ipséité) l'amène paradoxalement à s'engager dans une voie qui finalement relève plus du déterminisme familial (paroxysme d'une mêmeté socio-biographique) que de la prise d'autonomie.

Dans bien des cas, le projet personnel attendu par les conseillers d'orientation conduit finalement les élèves sur le chemin tracé par leur milieu familial, donnant corps à une tension équivalente à celle repérée entre " origine ouvrière et ambitions scolaires " (Brinbaum, Kieffer, 2009, p.567), qui témoigne de la difficulté à échapper à son appartenance sociale malgré le désir de réussite scolaire. Néanmoins, il arrive que certains fassent véritablement le choix d'une rupture. Semble alors se dessiner la possibilité de construire un projet véritablement personnel, qui n'est ni celui de l'institution scolaire, ni celui de l'entourage familial.

\section{2. Vides et trop pleins de la mémoire}

Pour comprendre de tels choix, il nous faut scruter le processus qui permet d'instaurer un point de rupture avec ce qui pouvait sembler être la voie toute tracée de leur destin. Nous allons voir qu'il se loge, en creux, dans les interstices de leur mémoire.

De manière générale, les souvenirs de la petite enfance, familiaux et scolaires, sont peu rapportés par les lycéens. Qu'il s'agisse d'éviter de se raconter ou d'un véritable oubli, cela révèle en creux le peu de place accordé à cet âge de la vie aux perspectives du présent et de l'avenir.

L'école maternelle reste toutefois un lieu dont les lycéens gardent de « bons " souvenirs. Les souvenirs d'école primaire, plus fréquents, sont également tenus à distance du présent. Les lycéens n'y voient que rarement le début de quelconques difficultés scolaires. Rares sont ceux qui envisagent d'ailleurs leur apparition, à un moment donné au cours de leur scolarité. Pour beaucoup, tout se passe comme si un niveau trop faible faisait irruption au moment de leur orientation en lycée, comme s'ils avaient soudain atteint une limite qui empêche leur poursuite d'études dans la filière choisie. Le passé apparaît ainsi dissocié des conditions de leur orientation au lycée.

Cette forme de désintérêt s'explique-t-elle par un rapport au temps différent ? Chez ces jeunes issus de milieux modestes, le temps semble ne pas se déployer de la même manière 
que chez ceux de milieux cultivés (Beaud, 1997), ce que B. Charlot (1999) voit d'ailleurs comme une raison de l'absurdité à vouloir leur faire élaborer un projet. Or, P. Ricoeur (1983) voit justement dans la possibilité de définir des intervalles de temps mesurables une condition de l'identité narrative.

Le récit de Gloria est à ce titre révélateur d'une histoire jalonnée de blancs. Inscrite en terminale Secrétariat, elle rêve de devenir danseuse. Comme bon nombre d'élèves, elle garde peu de souvenirs des débuts de sa scolarité. De la maternelle au CM1 : "Ça me fait un gros blanc dans ma mémoire". Au début du cycle d'école primaire, elle déménage avec sa mère et change d'établissement. Elle redouble son CE1 et change à nouveau d'école sans qu'elle puisse expliquer ces changements. Si l'histoire de Gloria est lacunaire, un jour reste en revanche parfaitement gravé dans sa mémoire. Il s'agit de celui où elle a dû partir chez son père :

"J'apprenais à monter à cheval et... quand j'ai vu mon papa avec deux policiers et ma grandmère, je me suis dit : “il y a un problème!" Et on m'a dit qu'ils venaient me chercher et qu'il $y$ avait un problème avec ma maman parce qu'elle s'était fait interner, euh... et mon père est venu me récupérer, donc, ils mont enlevée du centre. Je me souviens bien parce que j'adorais les chevaux et euh... on est rentré, on est allé chercher mes affaires et je suis retournée directement chez mon papa, et là je me suis dit "qu'est-ce qui s'est passé ? Pourquoi ?"”

Gloria se dit "vraiment choquée ». Le choc n’est toutefois pas lié à la prise de conscience violente des problèmes de sa mère, mais à l'interruption d'une activité, l'équitation, et aux conséquences de son déménagement. En arrivant chez son père, elle découvre autre chose : "Parce qu'après, j'ai appris pleins de nouvelles d'un coup, et après ça m'a, ça m'a bloquée quoi. Parce qu'au moment où je suis arrivée chez mon papa, je suis entrée dans l'entrée et je suis tombée sur une photo de mariage. Je l'ai regardée, j'ai fait: "tu t’es marié, papa?” (...). J'étais même pas invitée... et ben, j’en reviens pas!".

Sa mise à l'écart des recompositions familiales fait davantage violence que l'internement de sa mère. À la différence des lycéens évoqués précédemment, un souvenir a valeur d'événement véritable aux yeux de Gloria. En tant que tel, il est l'outil d'une mise en intrigue car " il est simplement l'inattendu, le surprenant, il ne devient partie intégrante de l'histoire que compris après coup, une fois transfiguré par la nécessité en quelque sorte rétrograde qui procède de la totalité temporelle menée à son terme " (Ricœur 1990, p. 170). Entre les blancs de la mémoire et des souvenirs trop vifs, Gloria élabore un récit de vie chaotique, désordonné, mais dans lequel les événements biographiques ont une forte portée affective.

Pas plus que Tarik, Gloria n'établit de lien entre ces deux histoires qui ne se croisent jamais. Elle aborde toutefois sa scolarité différemment. Si dans toutes les sociétés et selon des modalités très diverses, une dichotomie temporelle " avant/maintenant " organise toute remémoration (Candau, 1996, p. 39), elle n'intervient pas de la même manière dans les projets de Gloria et de Marine, cette dernière ayant dû s'extraire d'un cursus scolaire pour produire un regard rétrospectif et s'engager dans une autre voie. 
Rien de tel chez Gloria, toujours scolarisée en bac pro dans une filière sans aucun lien avec ses rêves de danseuse, mais bien résolue à obtenir son bac, envers et contre tout.

\section{3. Se rebeller contre le destin}

Pour Gloria, avoir son bac prend la forme d'une véritable rébellion : "Toutes mes søurs ont pas fait d'études. J'ai fait : moi je vais me rebeller, je vais faire des études! J'ai dit à mes parents: "J'ai eu mon BEP, cette année je vais essayer d'avoir le bac, je redouble jusqu'à avoir mon bac et si j'ai le bac, je ferai un BTS et je ferai tout pour avoir le BTS et si j'ai pas le BTS, je redouble jusqu'à temps d'avoir le BTS!" Mes parents ils me font: "Ouais, mais tu vas les finir à je sais pas quel âge les études! "Je fais : "Au moins, j’aurai eu mes diplômes!" À ce point-là mes parents ils me disent: "Tu es la seule des filles B. qui va continuer ses études!" "Gloria évoque ainsi sa fierté d'être arrivée en terminale alors qu'en $6^{\mathrm{eme}}$ son professeur principal, "qui devait pas beaucoup m'aimer", lui avait prédit l'échec. Le désamour, qui justifiait le rejet de l'école chez Tarik, est pour Gloria motif à révolte. C'est ainsi contre une forme de déterminisme familial et social plus large qui empêche les enfants de sa famille de "faire des études ", qu'elle choisit de lutter.

En conséquence, sa rébellion a pour corollaire une prise en main de sa destinée. Or, envisager son propre destin, c'est repérer "une trace, une marque qui est dans la famille et qui, comme la coutume, se transmet " (Verdier, 1995, p. 154). Le fatalisme qui pèse sur les enfants de la famille de Gloria et leur ferme la porte des études justifie ainsi son désir d'aller contre, impliquant l'articulation d'éléments propices à la linéarisation d'une histoire. Son récit met par conséquent en exergue les figures d'exception qui jalonnent son histoire : sa sœur, une amie inséparable, sa maîtresse de CM2, son professeur de chant, et son petit ami. En contrepoint, il y a les figures plus négatives : la demi-sœur avec laquelle elle ne s'entend pas, sa prof de $6^{\text {eme }}$ qui " ne l'aimait pas beaucoup ». C'est donc en termes affectifs que sont pensées les ressources d'une dynamique de projet personnel mais aussi les figures à combattre. Ces personnages apparaissent au final comme les moteurs de l'action. La mobilisation des ressources affectives, positives et négatives, lui permet de prendre son propre destin en main, promettant la "double conquête de la réussite sociale de son identité personnelle " (Ehrenberg, 1995, p. 15). Son récit se construit autour de l'idée d'un fil continu caché, comme à rebours, à travers l'ébauche d'une mise en lien des événements vécus. Aussi décousues soient-elles, des relations de cause à effet entre les éléments vécus y deviennent perceptibles. Cela permet à Gloria d'avoir des projets : obtenir son bac, faire de la danse classique, poursuivre ses études en BTS. Pour les concrétiser, elle met en œuvre des stratégies : elle gagne de l'argent en travaillant l'été, écrit des lettres de motivation et monte des dossiers pour obtenir un financement.

Cette expérience se déploie à travers une structuration narrative qui traduit une perception de l'identité non plus sous l'angle de la permanence, telle que nous l'avions envisagée en premier lieu, mais sous l'angle de l'ipséité, dans la mesure où les changements et les ruptures deviennent au contraire porteurs de sens et garants d'une continuité biographique. 


\section{Conclusion}

Rétablir la logique d'un cheminement biographique en tant que manière d'expliquer des ruptures, des événements familiaux, scolaires ou sociaux apparaît être une condition de la formulation d'un projet.

Les jeunes issus de milieux modestes qui se trouvent insidieusement dirigés vers des filières de baccalauréat professionnel voient leurs aspirations personnelles remises en cause. Ils composent alors de diverses manières avec les injonctions contradictoires qui leurs sont adressées à penser leur avenir. Ce rapport différencié à l'identité, théorisé par Paul Ricoeur, (1990) en référence aux notions "d’ipséité " qui implique la possibilité d'envisager des changements biographiques et d'une "mêmeté", ancrée au contraire dans une permanence de l'être, d'une identité immuable, s'ordonne autour de la capacité à entrer ou non en rébellion.

Ceux pour lesquels aucune prise de conscience des inégalités sociales n'affleure dans le discours tendent à se définir à partir de l'idée d'une identité substantielle, immuable dans le temps et indépendante de la mémoire (mêmeté). Si l'environnement familial, social et économique est difficile, il n'en demeure pas moins impossible à leurs yeux d'y voir une " cause " de déqualification scolaire. Ces lycéens acceptent alors avec une relative indifférence le rôle qu'on leur assigne dans la stratification scolaire. Les bouleversements affectifs rencontrés dans leur vie privée rendent insidieusement leur scolarité superfétatoire. Ils n'établissent aucune relation entre leur parcours scolaire et un contexte familial, économique, territorial, ce qui induit une forme de passivité vis-à-vis des injonctions scolaires au projet.

Ceux qui prennent conscience d'inégalités sociales pouvant expliquer leur relégation dans des filières de formation non choisies n'acceptent pas leur situation et tentent d'aller contre. Émerge alors une autre manière de penser le biographique (ipséité). Bien que les dés semblent jetés (Bourdieu, 1966), les jeunes s'arment pour " lutter » contre leur destin et s'ouvrir de nouveaux horizons en s'appuyant sur les ressources dont ils disposent. Le plus souvent, la rébellion se dirige contre l'institution scolaire, rendant un projet de formation institutionnel difficile à mettre en ouvre. Mais la rébellion prend parfois la forme d'une lutte contre un déterminisme social, et l'institution scolaire peut alors devenir l'allier d'un projet personnel.

La possibilité de répondre à l'injonction au projet apparaît, dans une large mesure, contredite par diverses contraintes, chez des jeunes dont la prise d'autonomie est réduite. Les lycéens se trouvent ainsi pris en porte à faux entre des logiques contradictoires : celles qui les somment de construire un projet professionnel personnel et celles qui les rendent dépendants du monde des adultes. Ce contexte induit un décalage entre les attentes de l'institution scolaire et ce que les lycéens en font : qu'ils se résignent ou qu'ils décident de prendre en main leur destin, ils se conforment finalement rarement aux injonctions institutionnelles. 
L'impasse de la construction d'un projet n'est donc pas à appréhender dans la seule conséquence du manque de disponibilité et de ressources concrètes et matérielles, mais dans ses fondements même. La structuration identitaire de ces jeunes détermine un rapport biographique et donc une logique du projet sans lien avec la manière dont l'institution scolaire et les adultes s'en font les porte-paroles. Ces modes de structuration identitaire au regard de l'injonction au projet gagneraient pourtant à être pris en compte par les acteurs éducatifs.

\section{- Bibliographie}

Arrigui J.-J., Gasquet C. (2010), «Orientation et affectation : la sélection dans l'enseignement professionnel du second degré ", Formation Emploi, n ${ }^{\circ} 109$, janvier-mars, pp. 99-112.

Arrigui J.-J., Sulzer E. (2012), "S’insérer à la sortie de l'enseignement secondaire : de fortes inégalités entre filières ", Bref-Céreq, n 303, décembre, pp. 1-4.

Beaud S. (1997), « Un temps élastique. Etudiants des « cités » et examens universitaires », Terrain, $n^{\circ} 29$, pp. 43-58.

Beaud S. (2002), $80 \%$ au bac... et après? Les enfants de la démocratisation scolaire, Paris, La Découverte.

Berthet T., Dechezelles S., Gouin R., Simon V. (2010), "La place des dynamiques territoriales dans la régulation de l'orientation scolaire“, Formation Emploi, n 109, janviermars, pp. 37-52.

Berthet T., Grelet Y., Romani C. (2008), Le système d'orientation : entre choix individuels et contraintes d'action publiques, $\mathrm{n}^{\circ} 36, \mathrm{NEF}$.

Bidart C. (2006), "Crises, décisions et temporalités : autour des bifurcations biographiques", Cahiers Internationaux de Sociologie, n¹20, pp. 29-57.

Biemar S., Philippe M.-C., Romainville M. (2003) «L'injonction au projet : paradoxale et infondée ?", OSP, n³2/1, pp. 31-51.

Borras I., Romani C. (2010), « Orientation et politiques publiques. Evolutions nationales, enjeux internationaux ", Formation Emploi, n 109, janvier-mars, pp. 9-22.

Boudesseul G. (2010), “La segmentation par l'orientation ? Comment l'orientation préfigure les inégalités d'emploi“, Formation Emploi, n 109, janvier-mars, pp. 53-70.

Boudesseul G., Grelet Y. (2010), “Choix des diplômes et projets de métiers selon l'environnement social local", Les Sciences de l'éducation-Pour l'ère nouvelle, n 2, Vol. 43, pp. 5-108. 
Bourdieu P. (1966), "Les inégalités devant l'école et devant la culture ", Revue Française de Sociologie, pp. 325-347.

Boutinet J. P. (2005), Anthropologie du projet, Paris, PUF.

Brinbaum Y., Guégnard C. (2012), « Parcours de formation et d'insertion des jeunes issus de l'immigration au prisme de l'orientation ", Formation Emploi, n ${ }^{\circ} 118$, avril-juin, pp. 61-82.

Brinbaum Y., Kieffer A. (2009), "Les scolarités des enfants d'immigrés de la sixième au baccalauréat : différenciation et polarisation des parcours ", Population, 64, 3, pp. 561-610.

Candau J. (1996), Anthropologie de la mémoire, Paris, PUF.

Charlot B. (1999), Le Rapport au Savoir en milieu populaire. Une recherche dans les lycées professionnels de banlieue, Paris, Anthropos.

Coineau C., Vivent C. (2010), « Les orientations scolaires, entre tâtonnement et réappropriation ", Formation Emploi, n' 109 , janvier-mars, pp. 71-84.

Coquelle C. (1994), "Attention projet! ", Formation Emploi, n 45, janvier-mars, pp. 25-32.

Daverne C. (2012), «De l'expérimentation à la généralisation du baccalauréat professionnel en trois ans (BP3) Parcours d'élèves (dés) enchantés ", Education et francophonie, $\mathrm{n}^{\circ} 1$, pp. 198-212.

Douat E. (2011), L'école buissonnière, Paris, La Dispute.

Duru-Bellat M., Minga A., (1993), Pour une approche analytique du fonctionnement du système éducatif, Paris, PUF.

Duru-Bellat M. (2002), Les inégalités sociales à l'école : genèses et mythes, Paris, PUF.

Duru-Bellat M., Kieffer A. (2008), « Du baccalauréat à l'enseignement supérieur : déplacement et recomposition des inégalités ", Population, pp. 123-158.

Ehrenberg A. (1995), Lindividu incertain, Paris, Hachette.

Esterle-Hedibel M, (2007), Les élèves transparents. Les arrêts de scolarité avant 16 ans, PU du Septentrion, coll. " Métiers de la formation ».

Fournier G. (2002), «L’insertion socioprofessionnelle: vers une compréhension dynamique de ce qu'en pensent les jeunes ", Numéro spécial. Revue internationale de Carriérologie, 8(3), pp. 365-387.

Geminel P. (1988), "Les jeunes en stage " 16-18 ans » : une approche des attitudes en termes de projet ", Revue française de sociologie. $n^{\circ} 29-1$, pp. 143-170. 
Grelet Y. (2006), «Des territoires qui façonnent les parcours scolaires des jeunes », BrefCéreq. n 228, mars,

Insee (2013), BIT France-décembre 2013.

Lahire B. (2001), L'homme pluriel. Les ressorts de l'action, Paris, Nathan.

Lahire B. (2005), L’esprit sociologique, Paris, La Découverte, coll. «Textes à l'appui ».

Landrier S., Nakhili N. (2010), «Comment l'orientation contribue aux inégalités de parcours scolaires en France ", Formation Emploi, n 109, janvier-mars, pp. 23-35.

Lévi-Strauss C. (1962), La pensée sauvage, Paris, Plon.

Merle P. (2002), La démocratisation de l'enseignement, Paris, La Découverte.

Pémartin D., Legrès J. (1988), Les projets chez les jeunes. La Psychopédagogie des projets personnels, Issy-les-Moulineaux, EAP.

Prost A. (1986), L'enseignement s'est-il démocratisé?, Editions du Scarabée.

Rey A, (1995) Dictionnaire historique de la langue française, Paris, Dictionnaire Le Robert.

Ricoeur P. (1983), Temps et récit; 1 L’intrigue et le récit historique, Paris, Seuil.

Ricoeur P. (1990), Soi-même comme un autre, Paris, Seuil.

Van Zanten A. (2001), L'école de la périphérie. Scolarité et ségrégation en banlieue, Paris, Coll. « Le lien social », PUF.

Verdier Y. (1995), Coutume et destin, Thomas Hardy et autres essais, Paris, Gallimard. 


\section{TRAVAIL et EMPLOI}

\section{UNE CRISE SANS PRÉCÉDENT ? EXPÉRIENCES ET CONTESTATIONS DES RESTRUCTURATIONS (2)}

- Carrières de licencié.e.s. Revenir sur le passé pour saisir les destins des chômeurs de Moulinex

- La déstabilisation des stables : restructuration financière et travail insoutenable

- Au-delà de l'emploi, quelle sauvegarde du travail ?

Expériences du reclassement dans le cadre d'un PSE « idéal »

- Les restructurations, entre opportunités et contraintes : reconversion et création d'entreprise dans le secteur des énergies renouvelables

- La lutte, et après ? Une association de salariés licenciés, entre mobilisations collectives et action sociale

- Crise du travail et redéfinition des frontières du social en Argentine à travers le prisme de la mobilisation des desocupados

- «Militer ça donne des forces. »Potentiel transformateur et intégrateur de l'action collective des chômeurs

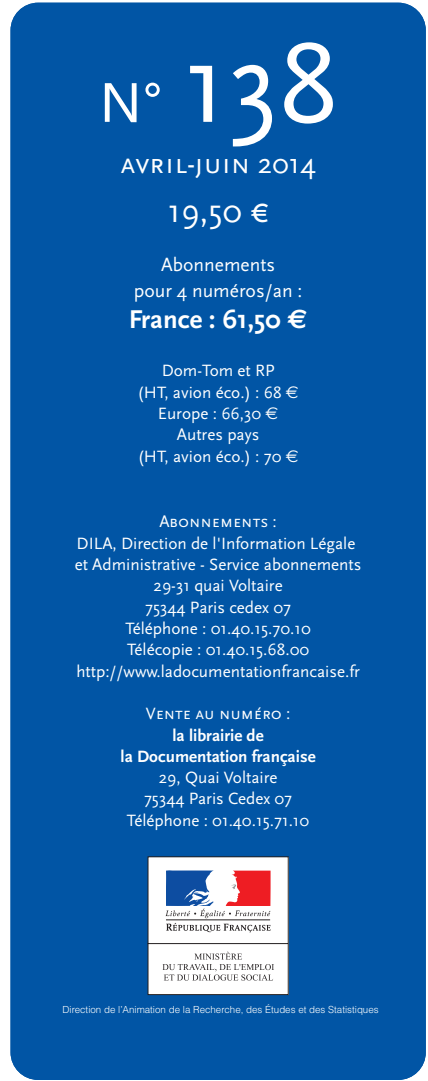$$
\text { et intégrateur de laction collective des chomeurs }
$$ 